\title{
Analysis of Artifact with X-ray CT Using Energy Band by Photon Counting CdTe detector
}

\author{
G.Matsumoto $^{\text {a,b, }}{ }^{*}$, Y.Imura $^{\mathrm{a}}$, H.Morii ${ }^{\text {a }}$, A.Miyake ${ }^{\text {a }}$, T.Aoki ${ }^{\text {a }}$ \\ ${ }^{a}$ Research Institute of Electronics, Shizuoka University, Hamamatsu 432-8011, Japan \\ ${ }^{\mathrm{b}}$ Department of Bioengineering, Toledo University, Toledo, OH 43606-3390, USA
}

\section{Abstract}

This report analyzes the imaging errors in what are called "artifact" by CdTe detector with the energy differentiation ability using the photon counting mode. The selection of the specific energy level in each metal sample allows nominating the images with less artifact. In addition, the inspection of $\mu x$ is the supportive method to judge the energy level. Photon counting CdTe detector with the energy differentiation ability is a system what can provide the information of projection data at different energy bands to establish the accuracy image of any materials.

Keywords: X-ray, CT, energy band, artifact, photon counting, CdTe detector

\section{Introduction}

$\mathrm{X}$-ray $\mathrm{CT}$ is a nondestructive technique for visualizing characteristics in the interior of subjects, and for obtaining the digital information on their 3-D geometries and other properties. This system is the crucial inspection device for many varieties of material such as rocks, metals, ceramic and soft tissues. Consequently, X-ray CT is widely used in a variety of fields such as the medical, security, and industry fields.

X-ray CT imaging system is fundamentally counted on by the detector performance. CT images should be acquired without any imaging errors. For example, medical diagnosis is carried out with CT image to detect any diseases. In order to diagnose more efficiently, the X-ray detector has a crucial role on capturing the images. Currently, the cadmium telluride (CdTe) detector is suggested to utilize as a radiation detector. CdTe is a suited material for hard X-ray imaging spectrometers because it has a high detection efficiency[1] and good energy resolution comparable to that of conventional detectors. In

\footnotetext{
* Genki Matsumoto Tel: +81-53-478-1321 E-mail: genki.matsumoto@utoledo.edu
} 
addition, CdTe detector can measure X-ray energy by photon-counting. The photon absorbed in the detector results in creating the electron-hole pair by interaction in materials. The energy of the X-ray can be measured by the number of charges one photon can generate. Since the detector counts the number of charges, it generates the high accuracy image of the material comparable to the image obtained by a conventional X-ray detector.

However, X-ray CT usually has a disadvantage in terms of the quality of the images, which is called artifact [1]. In the procedure of capturing the cross-section image, these imaging errors occur. When the sinogram is acquired, it contains the defect data from beam hardening effect. Then, this data establishes the shape of what is different from the actual shape on image by reconstruction. This unexpected data causes artifact resulting in inaccuracy image. Artifact results in the blurry and obscure images of materials. Beam hardening effect is also a major imaging error when metal samples are scanned $[2,3]$. It is known that when beam hardening effect is taking place on metal sample images, the more artifact can be contained on it. To avoid these imaging errors, the energy differentiation ability in CdTe detector plays a significant role[4]. The energy differentiation by CdTe detector while scanning metal samples brings the selection of images on which artifact does not give an effect. In fact, the artifact is unable to be reduced by this ability, the selection of the certain energy bands are the key to indicate the image contained less imaging errors. The energy differentiation ability allows inspecting variety of images captured at each energy level. Therefore, photon-counting mode by CdTe detector with the energy differentiation ability can be the ideal imaging system for establishment of three - dimensional measurements with the accurate images.

\section{Experiment}

The experiment was executed by scanning samples with 64-channel CdTe radiation sensor (Hamamatsu) which consisted of 64 elements aligned side by side at intervals of $0.1 \mathrm{~mm} \mathrm{[5,6].} \mathrm{X-ray} \mathrm{is}$ given off from the X-ray tube (150kV Micro Focus X-ray tube L8121). The samples were located and rotated between the X-ray tube and CdTe detector as X-ray was given off. The constant rotation speed and time of scanning were set to be $2 \%$ and $180 \mathrm{~s}$. The samples were placed at $527 \mathrm{~mm}$ from the X-ray tube and $290 \mathrm{~mm}$ from CdTe detector. After sinogram was built, CT images could be established by reconstruction, which is called the back projection filtered method [7]. Metal samples inspected in this experiment were the $10 \mathrm{~mm} \times 10 \mathrm{~mm}$ square-shape with $5 \mathrm{~mm}$ hole in diameter of aluminum, iron, copper, molybdenum, and the $15 \mathrm{~mm}$ diameter cylindrical-shape with $6 \mathrm{~mm}$ hole in diameter of nickel for each energy band with $10 \mathrm{keV}$ width from 40 to $130 \mathrm{keV}$ in $\mathrm{X}$-ray photon energy. 


\section{Results and Discussion}

The beam hardening effect was systematically investigated and shown in Figure 1 (a). It describes the differential of X-ray attenuation coefficient (described as $\mu_{\mathrm{CT}}$ below) in the metal region at each energy level which the differential of the $\mu_{\mathrm{CT}}$ was derived from the method. Shown in Figure 1 (b), which indicates the $\mu_{\mathrm{CT}}$ values of molybdenum at $100 \mathrm{kev}$, the circled region are regarded as the region where beam hardening effect had taken place since $\mu_{\mathrm{CT}}$ is ideally kept at the constant value in the metal region. The presence of visible curve in the circled region shows beam hardening effect which makes the images blurry and inaccurate. The two critical points where give the maximum gap in metal region were easily identified. The channels corresponding to these two points were determined to seek the $\mu_{\mathrm{CT}}$ value at each energy level. Calculation of differential of $\mu_{\mathrm{CT}}$ between the two was executed to show Figure 1 (a) for understanding how the differential changes as the energy level increases. Figure 1 (a) shows that $\mu_{\mathrm{CT}}$ of all metal samples, excluding aluminum, tend to decrease slightly as energy level gets higher. It was obvious that the differential at each energy level of aluminum sample had no visible change, which describes that beam hardening effect cannot be seen on this sample since aluminum has lower atomic mass and X-ray easily passes through it. However, it finds out that $\mu_{\mathrm{CT}}$ of iron and copper sample was getting lower and eventually reached the constant value of $\mu_{\mathrm{CT}}$ over $80 \mathrm{keV}$ to $90 \mathrm{keV}$, which shows that around this energy band is an ideal energy level to establish the high quality image without being influenced by beam hardening effect. The trend lines of iron and copper were close to each other because same shaped-samples were used and these are aligned in the periodical table. In molybdenum sample case, beam hardening effect appeared till $80 \mathrm{keV}$. However, it is obvious to mention that scanning with the higher energy band can result in the image with the lower influence from beam hardening effect. The investigation about the data of nickel sample is described in terms of the relationship with the width below.

\section{Figure1.}

Artifact in the CT image is focused on the outside of metal samples. Figure 1 (c) shows the integral of $\mu_{\mathrm{CT}}$ value in the region of outside of sample at each energy level. The calculation of the integral was carried out by the following procedure. The width of the metal sample can be defined with the points where the line with the higher slope intersects at $\mathrm{x}$-axis. The region outside of the metal sample is now able to be inspected as the region where artifact occurred. The $\mu_{\mathrm{CT}}$ values at each channel outside of sample area are summed up and compared. The $\mu_{\mathrm{CT}}$ outside of the metals are usually close to zero under the ideal simulation. Therefore, the summation of $\mu_{\mathrm{CT}}$ can be investigated to understand how much artifact contains in outside of metal area. Shown in Figure 1(c), the integral of $\mu_{\mathrm{CT}}$ was clearly 
decreased as energy level gets higher. It also describes that the higher the sample's atomic mass, the more artifact gives the effect on the image at the certain energy level. As mentioned in beam hardening effect results, $\mu_{\mathrm{CT}}$ of aluminum sample had the same results. The trend lines of other metal samples were similar in terms of the decrease of $\mu_{\mathrm{CT}}$ as energy increases. So that, Figure 1(c) can describe that scanning with the high energy band brings the more accuracy image if the sample with higher atomic mass need to be captured. Figure 2(a) represents the CT image of molybdenum sample at $40 \mathrm{keV}, 80 \mathrm{keV}$, and $120 \mathrm{keV}$ and Figure 2(b) shows the shape with dimension. Figure 2 emphasizes that the energy differentiation is significantly helpful for the creation of the less-noise image.

Figure 2.

Results from the estimation of beam hardening effect and artifact show that the utilization of high energy band is one of the most essential scanning methods, since the higher the energy band is used, the less imaging errors are taking place. It proves that the higher energy band can be effective to generate the less-error image. However, it is difficult to judge which ideal energy level must be adjusted to create the minimum errors on image in each metal sample because each metal has a different energy level where beam hardening effect or artifact are taken place as less as possible. To predict the specific energy level for each metal or sample, $\mu x$ is the essential matter for judgment. Each atom possesses a particular $\mu$ value (unit is $\mathrm{cm}^{-1}$ ) and it is theoretically known that the atom with higher atomic mass has the higher $\mu$ value. The relationship between intensity and $\mu$ is shown as

$$
I=I_{0} \exp (-\mu x)
$$

where $I$ and $I_{0}$ are the intensity before and after X-ray passes through the sample. The width of sample $(x)$ is the matter to be considered when the sinogram is captured. Therefore, the value on the sinogram is shown as $\mu x$. In addition, $\mu_{\mathrm{CT}}$ is derived from elimination of the width sample in the process of creating CT image. Before reconstruction to generate a CT image, the investigation of $\mu x$ in each metal sample is important when the energy level needs to be identified because the data of $\mu_{\mathrm{CT}}$ is derived from $\mu x$ value. Shown in Figure 1(a), nickel sample had the differential of $\mu_{\mathrm{CT}}$ more than the copper sample even though nickel has less atomic mass because the size of nickel sample is thicker $(15 \mathrm{~mm})$ than that of the copper sample $(10 \mathrm{~mm})$. If the nickel sample needs to have the same accuracy of image as the copper sample, the energy level for the nickel should be higher than copper sample in order to be used. $\mu x$ value can be the clue to estimate the energy level for metal samples.

In conclusion, CdTe detector with the energy differentiation ability can open a new path for Xray applications, in terms of the quality of CT images. Utilization of X-ray tube and 64-channel CdTe 
detector allows measuring the projection data at each energy level simultaneously. As a result, the investigation of quality of image can be performed at each energy level. It is certain that beam hardening effect hardly occurs as an energy level increases. The energy level where the imaging errors are getting less on each metal is uncertain, therefore, $\mu x$ value should be paid attention to judge which energy level describes the image with the less beam hardening effect and artifact. Higher energy level comes with the lower $\mu x$ value, which means that the image is not influenced by beam hardening effect and artifact. Consequently, the energy differentiation ability is the essential inspection method guaranteed to provide the higher quality image.

Fig. 1. (Color online) Investigation of the imaging errors of metal samples. $\mathrm{Al}, \mathrm{Fe}, \mathrm{Cu}, \mathrm{Mo}$ : $10 \mathrm{~mm} \mathrm{x}$ $10 \mathrm{~mm}$ square-shape, Ni: cylindrical-shape with $\phi=15 \mathrm{~mm}$. (a) Detail of region where the calculation executed for beam hardening effect. (b) Beam hardening effect in the metal region. (c) Artifact in the outside of the metal region.

Fig. 2. (Color online) (a) CT images of molybdenum at $40 \mathrm{keV}, 80 \mathrm{keV}$, and $120 \mathrm{keV}$. (b) Molybdenum sample detail with dimension.

[1] G. Chiro, R. Brooks, L. Dubal, E.Chew, Journal of Computer Assisted Tomography. 2, Issue 1 (1978).

[2] W. Zou, T. Nakashima, Y. Onishi, A. Koike, B. Shinomiya, H. Morii, Y. Neo, H. Mimura, T. Aoki, Japanese Journal of Applied Physics. 47, 7317-7323 (2008).

[3] R. Brooks, G. Chiro, Phys. Med. Biol. 21, 390-398 (1976).

[4] T. Nakashima, H. Morii, Y. Neo, H. Mimura, T. Aoki, Proc. SPIE. 6706, 67060C (2007).

[5] Y. Tomita, Y. Shirayanagi, S.Matsui, T. Aoki, Y. Hatanaka, Proc. of SPIE. 5922, 59220A (2005)

[6] Y. Ohno, M. Torikoshi, T.Tsunoo, Nuclear Instruments and Methods in Physics Research. A 548, 72 77 (2005).

[7] R. Brooks, G. Chiro, Phys. Med. Biol. 21, 689-732 (1976). 

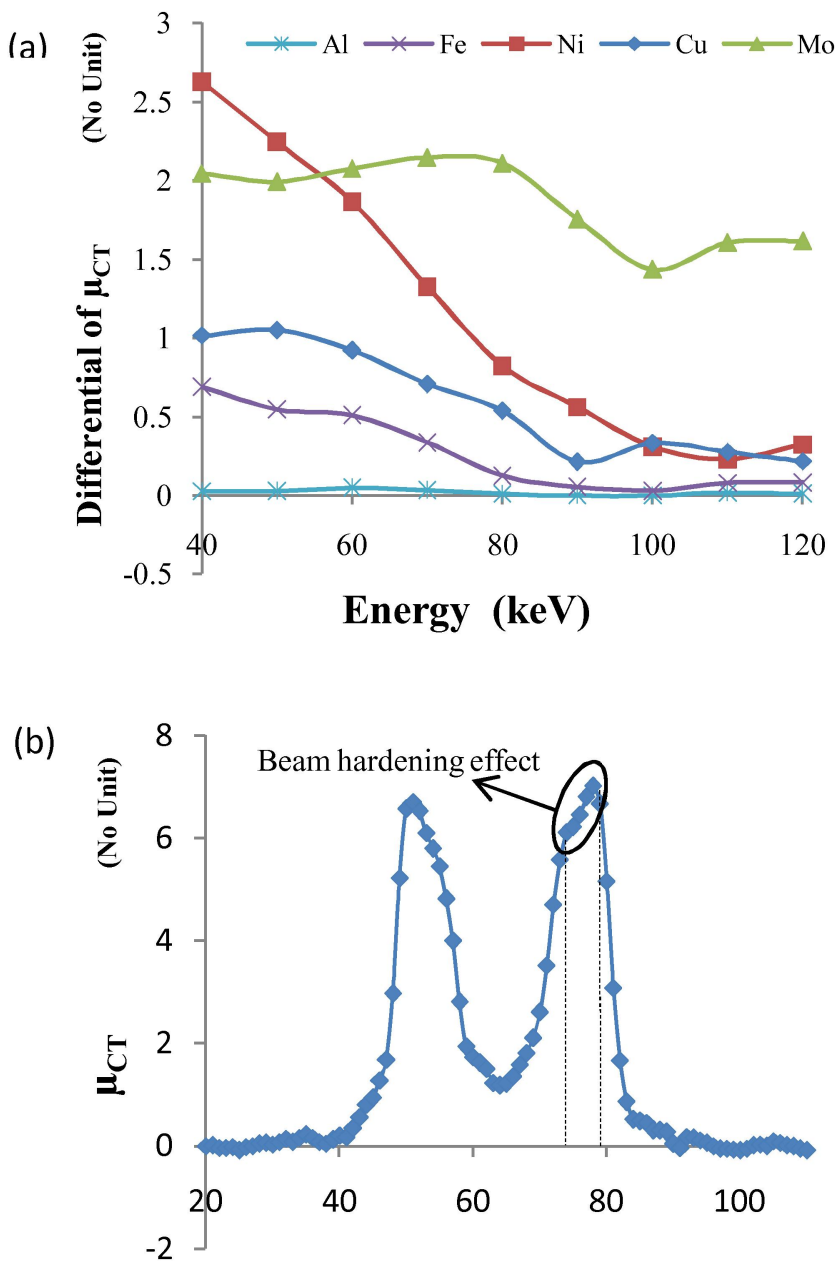

\section{Pixel}

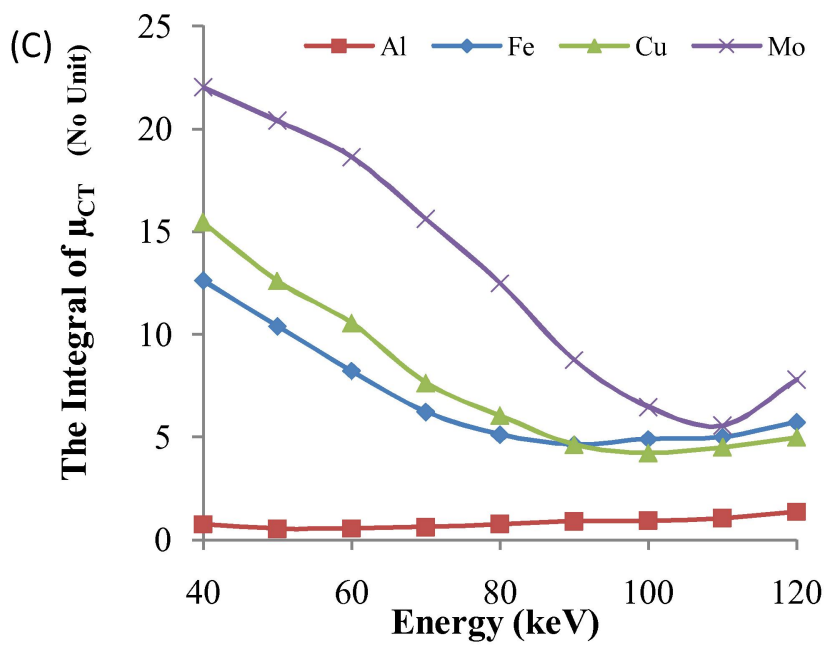




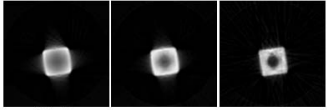
$40 \mathrm{keV}$

(b)

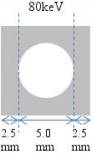

$120 \mathrm{keV}$ 